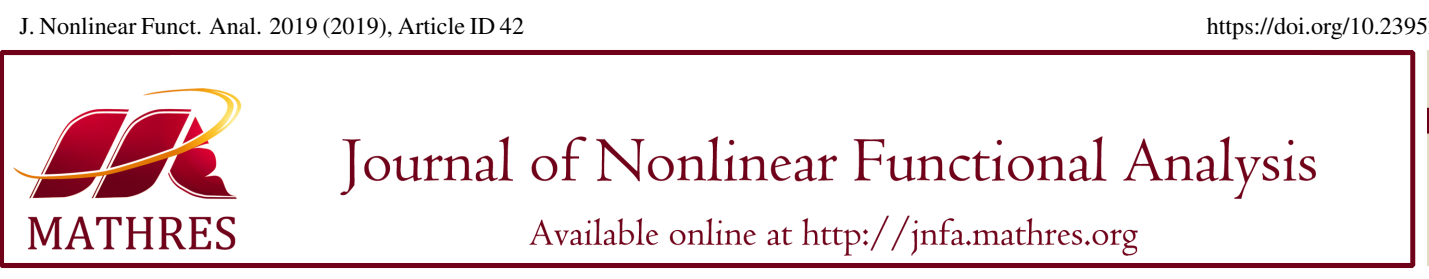

https://doi.org/10.23952/jnfa.2019.42

\title{
NONTRIVIAL SOLUTIONS FOR PARTIAL DISCRETE DIRICHLET PROBLEMS VIA A LOCAL MINIMUM THEOREM FOR FUNCTIONALS
}

\author{
SHAPOUR HEIDARKHANI ${ }^{1}$, MAURIZIO IMBESI ${ }^{2, *}$ \\ ${ }^{1}$ Department of Mathematics, Faculty of Sciences, Razi University, 67149 Kermanshah, Iran \\ ${ }^{2}$ Department of Mathematical and Computer Sciences, Physical and Earth Sciences, University of Messina, \\ Viale F. Stagno d'Alcontres 31, 98166 Messina, Italy
}

\begin{abstract}
Based on a local minimum theorem for differentiable functionals, the existence of non-trivial solutions for a partial discrete Dirichlet problem depending on a real parameter is discussed.

Keywords. Discrete nonlinear boundary value problems; Algebraic systems; Nontrivial solutions; Difference equations, Critical points theory.
\end{abstract}

2010 Mathematics Subject Classification. 39A10, 34B15, 35B38.

\section{INTRODUCTION}

The aim of this paper is to establish existence results for the discrete system, namely, $\left(E_{\lambda}^{f}\right)$, given as follows

$$
\begin{gathered}
{[u(i+1, j)-2 u(i, j)+u(i-1, j)]+[u(i, j+1)-2 u(i, j)+u(i, j-1)]} \\
+\lambda f((i, j), u(i, j))=0, \quad \forall(i, j) \in[1, m] \times[1, n]
\end{gathered}
$$

with boundary conditions

$$
\begin{gathered}
u(i, 0)=u(i, n+1)=0, \quad \forall i \in[1, m], \\
u(0, j)=u(m+1, j)=0, \quad \forall j \in[1, n],
\end{gathered}
$$

where $[1, m]=\{1, \cdots, m\},[1, n]=\{1, \cdots, n\}, f:[1, m] \times[1, n] \times \mathbb{R} \rightarrow \mathbb{R}$ denotes a continuous function and $\lambda$ is a positive real parameter.

In recent years, a great deal of work has been done in the study of the existence of solutions for nonlinear algebraic systems for discrete boundary value problems due to applications such as the discrete models of steady-state equations of reaction-diffusion equations, the steady-state equations of coupled map lattices, the steady-state equations of compartmental system, the steady-state models on complex

${ }^{*}$ Corresponding author.

E-mail addresses: sh.heidarkhani@razi.ac.ir (S. Heidarkhani), imbesim@unime.it (M. Imbesi).

Received July 11, 2019; Accepted September 12, 2019.

(C)2019 Journal of Nonlinear Functional Analysis 
dynamical network, and the steady-state systems of discrete Turing instability models (see [1] and the references therein). Discrete problems involving functions with two or several discrete variables are very relevant and have been investigated deeply. Such an active interest is undoubtedly due to the advance of modern digital computing devices. Indeed, since these relations can be simulated in a relatively easy manner by means of these devices and since such simulations often reveal important information about the behavior of complex systems, a large number of recent investigations related to image processing, population models, artificial or biological neural networks, social behaviors, digital control systems, computer science and mechanical engineering were described. In order to establish the existence and multiplicity of solutions for discrete problems under some restrictions on $f$ (sublinear or superlinear at infinity), several authors exploited various abstract methods as fixed point theorems, Brouwer degree and critical point theory; see, for instance, $[2,3]$ and $[4,5,6]$ for related topics. We also cite the papers $[6,7,8,9,10,11,12,13,14,15,16]$ for some interesting contributions related to the existence results for nonlinear algebraic systems, as well as the books [17] and [18] as general references for discrete problems. For example, Zhang and Liu [6] dealt with a class of semipositone discrete boundary value problems via critical point theory, and obtained nonexistence and multiplicity results on sublinear nonlinearities and an existence result on superlinear nonlinearities, respectively. Zhang and Feng [16], applying fixed point theorems, proved results on existence, uniqueness, multiplicity and nonexistence of positive solutions for a class of nonlinear algebraic system. Ji and Yang [10] studied the structure of the spectrum of the problem $\left(E_{\lambda}^{f}\right)$ and investigated the existence of a positive eigenvector corresponding to the smallest eigenvalue. Galewski and Orpel [7], through variational methods and by using some monotonicity results, analyzed problem $\left(E_{\lambda}^{f}\right)$ and obtained the existence of one solution. Moreover, Kristály, Mihăilescu and Rădulescu [19] studied a discrete boundary value problem, where the nonlinear term has an oscillatory behaviour near the origin or at infinity. In fact, by a direct variational method, they showed that the problem has a sequence of non-negative, distinct solutions which converges to 0 (resp. $+\infty$ ) in the sup-norm whenever the nonlinear term oscillates at the origin (resp. at infinity). In [20], Kristály et al. studied an eigenvalue problem in the framework of difference equations and showed that there exist two positive constants $\lambda_{0}$ and $\lambda_{1}$ verifying $\lambda_{0} \leq \lambda_{1}$ such that any $\lambda \in\left(0, \lambda_{0}\right)$ is not an eigenvalue of the problem, while any $\lambda \in\left[\lambda_{1},+\infty\right)$ is an eigenvalue of the problem, and they gave some estimates for $\lambda_{0}$ and $\lambda_{1}$. In [21], Cabada, Iannizzotto and Tersian extended a recent multiplicity theorem for the critical points of a functional defined on a finitedimensional Hilbert space due to Ricceri, and employed their result to discuss the existence of at least three solutionsfor Dirichlet boundary value problems for difference equations involving the discrete $p$-Laplacian operator. In [11], Molica Bisci and Repovš, using a multiple critical points theorem for locally Lipschitz continuous functionals, established the existence of at least three distinct solutions for a parametric discrete differential inclusion problem involving a real symmetric and positive definite matrix. They presented also applications to tridiagonal, fourth-order and partial difference inclusions. In [9], employing a version of [22, Theorem 2.1], the existence of infinitely many solutions for the discrete Dirichlet problem $\left(E_{\lambda}^{f}\right)$ was examined. More precisely, unbounded intervals of parameters such that the problem $\left(E_{\lambda}^{f}\right)$ admits either an unbounded sequence of solutions, provided that the nonlinearity has a suitable behaviour at infinity, or a pairwise distinct sequence of solutions that strongly converges to zero if a similar behaviour occurs at zero have been determined. Based on variation methods and critical point theory, Heidarkhani and Imbesi [8] established the existence of multiple solutions for $\left(E_{\lambda}^{f}\right)$. More precisely, under appropriate assumptions 
on the nonlinearities, they determined exact collections of parameters such that the problem admits at least three solutions.

In this paper, using two consequences of a local minimum theorem for differentiable functionals [23], we discuss the existence of at least one nontrivial solution for the problem $\left(E_{\lambda}^{f}\right)$.

For an overview on this subject, we refer the reader to the papers $[4,24]$. See, for more details about partial difference equations, the monograph of Cheng [25].

\section{NOTATIONS AND BASIC FACTS}

Let $(X,\|\cdot\|)$ be a finite dimensional Banach space and let $J_{\lambda}: X \rightarrow \mathbb{R}$ be a function satisfying the following structure hypothesis:

(A) $J_{\lambda}(u):=\Phi(u)-\lambda \Psi(u)$ for all $u \in X$, where $\Phi, \Psi: X \rightarrow \mathbb{R}$ are two functions of class $C^{1}$ on $X$ with $\Phi$ coercive, i.e. $\lim _{\|u\| \rightarrow \infty} \Phi(u)=+\infty$, and $\lambda$ is a real positive parameter.

In this framework finite dimensional variants of [23, Theorem 5.1] and [23, Theorem 5.3] are the following theorems, respectively: the consequences of the existence results of a local minimum [23, Theorem 3.1] which are inspired by Ricceri's variational principle (see [26]).

We define the following functions

$$
\begin{gathered}
\vartheta\left(r_{1}, r_{2}\right)=\inf _{v \in \Phi^{-1}\left(r_{1}, r_{2}\right)} \frac{\sup _{u \in \Phi^{-1}\left(r_{1}, r_{2}\right)} \Psi(u)-\Psi(v)}{r_{2}-\Phi(v)}, \\
\rho_{1}\left(r_{1}, r_{2}\right)=\sup _{v \in \Phi^{-1}\left(r_{1}, r_{2}\right)} \frac{\Psi(v)-\sup _{u \in \Phi^{-1}\left(-\infty, r_{1}\right]} \Psi(u)}{\Phi(v)-r_{1}}
\end{gathered}
$$

for all $r_{1}, r_{2} \in \mathbb{R}, r_{1}<r_{2}$, and

$$
\rho_{2}(r)=\sup _{v \in \Phi^{-1}(r,+\infty)} \frac{\Psi(v)-\sup _{u \in \Phi^{-1}(-\infty, r]} \Psi(u)}{\Phi(v)-r}
$$

for all $r \in \mathbb{R}$.

Lemma 2.1. Assume that there are $r_{1}, r_{2} \in \mathbb{R}, r_{1}<r_{2}$ such that

$$
\vartheta\left(r_{1}, r_{2}\right)<\rho_{1}\left(r_{1}, r_{2}\right) .
$$

Setting $I_{\lambda}:=\Phi-\lambda \Psi$, for each $\left.\lambda \in\right] \frac{1}{\rho_{1}\left(r_{1}, r_{2}\right)}, \frac{1}{\vartheta\left(r_{1}, r_{2}\right)}\left[\right.$, there is $u_{0, \lambda} \in \Phi^{-1}\left(r_{1}, r_{2}\right)$ such that $I_{\lambda}\left(u_{0, \lambda}\right) \leq I_{\lambda}(u)$ $\forall u \in \Phi^{-1}\left(r_{1}, r_{2}\right)$ and $I_{\lambda}^{\prime}\left(u_{0, \lambda}\right)=0$.

Lemma 2.2. Fix $\inf _{X} \Phi<r<\sup _{X} \Phi$ and assume that

$$
\rho_{2}(r)>0
$$

and for each $\lambda>\frac{1}{\rho_{2}(r)}$, the functional $I_{\lambda}:=\Phi-\lambda \Psi$ is coercive. Then for each $\left.\lambda \in\right] \frac{1}{\rho_{2}(r)}$, $+\infty[$, there is $u_{0, \lambda} \in \Phi^{-1}(r,+\infty)$ such that $I_{\lambda}\left(u_{0, \lambda}\right) \leq I_{\lambda}(u) \forall u \in \Phi^{-1}(r,+\infty)$ and $I_{\lambda}^{\prime}\left(u_{0, \lambda}\right)=0$.

As ambient space $X$, we consider the $m n$-dimensional Banach space $\mathbb{R}^{m n}$ endowed by the norm

$$
\|u\|:=\left(\sum_{k=1}^{m n} u_{k}^{2}\right)^{\frac{1}{2}} .
$$

Moreover, $\mathfrak{M}_{\imath \times \imath}(\mathbb{R}), \imath \in \mathbb{N}$, stands for the linear space of all the matrices of order $\imath$ with real entries. Let $h:[1, m] \times[1, n] \rightarrow[1, m n]$ be the bijection defined by $h(i, j):=i+m(j-1)$, for every 
$(i, j) \in[1, m] \times[1, n]$. As pointed out in [9], it can be displayed in the following way:
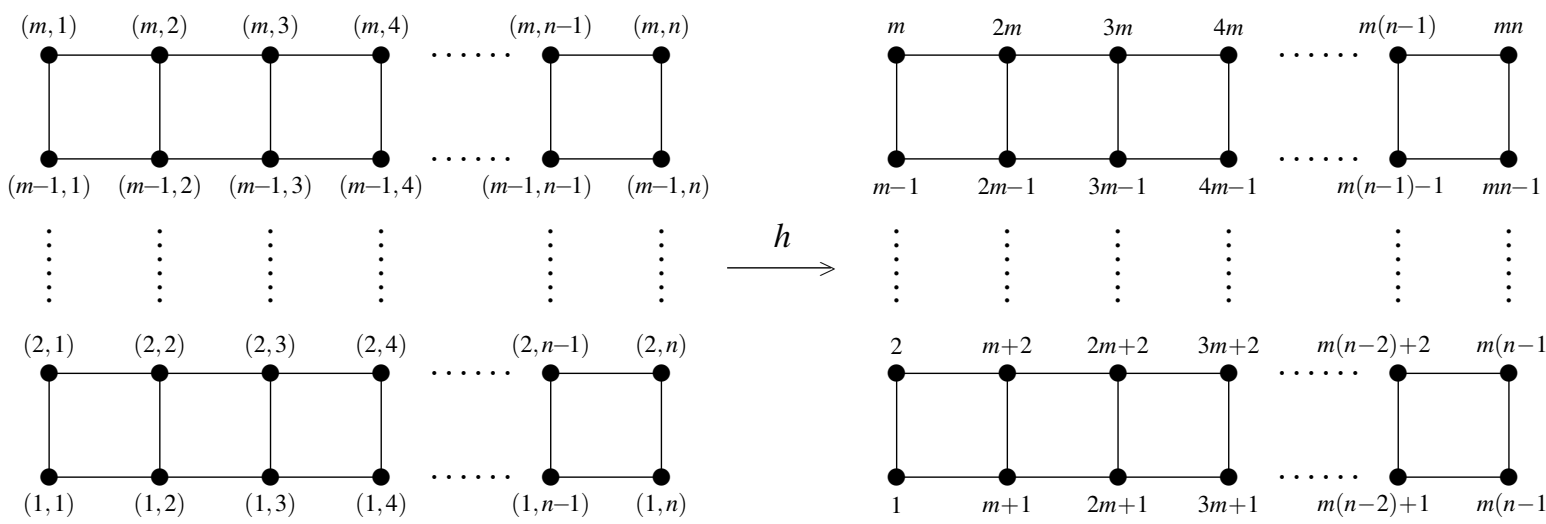

Let's denote $w_{k}:=u\left(h^{-1}(k)\right)$ and $g_{k}\left(w_{k}\right):=f\left(h^{-1}(k), w_{k}\right)$, for every $k \in[1, m n]$.

Thus, problem $\left(E_{\lambda}^{f}\right)$ can be written as the nonlinear algebraic system

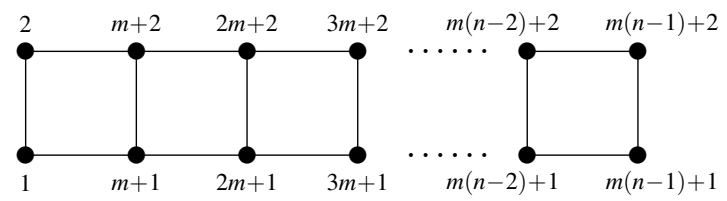

$$
A w=\lambda g(w),
$$

in which $A:=\left(a_{i j}\right)$ is the matrix $\left(\begin{array}{ccccccc}L & -I & 0 & \ldots & 0 & 0 & 0 \\ -I & L & -I & \ldots & 0 & 0 & 0 \\ 0 & -I & L & \ldots & 0 & 0 & 0 \\ 0 & 0 & -I & \ldots & 0 & 0 & 0 \\ & & & \ddots & & & \\ 0 & 0 & 0 & \ldots & -I & 0 & 0 \\ 0 & 0 & 0 & \ldots & L & -I & 0 \\ 0 & 0 & 0 & \ldots & -I & L & -I \\ 0 & 0 & 0 & \ldots & 0 & -I & L\end{array}\right) \in \mathfrak{M}_{m n \times m n}(\mathbb{R})$

such that $L$ is the submatrix $\left(\begin{array}{ccccccc}4 & -1 & 0 & \ldots & 0 & 0 & 0 \\ -1 & 4 & -1 & \ldots & 0 & 0 & 0 \\ 0 & -1 & 4 & \ldots & 0 & 0 & 0 \\ 0 & 0 & -1 & \ldots & 0 & 0 & 0 \\ & & & \ddots & & & \\ 0 & 0 & 0 & \ldots & -1 & 0 & 0 \\ 0 & 0 & 0 & \ldots & 4 & -1 & 0 \\ 0 & 0 & 0 & \ldots & -1 & 4 & -1 \\ 0 & 0 & 0 & \ldots & 0 & -1 & 4\end{array}\right) \in \mathfrak{M}_{m \times m}(\mathbb{R})$,

$I \in \mathfrak{M}_{m \times m}(\mathbb{R})$ is the identity matrix, and $g(w):=\left(g_{1}\left(w_{1}\right), \ldots, g_{m n}\left(w_{m n}\right)\right)^{t}$, for every $w \in X$.

Moreover, a direct computation ensures that

$$
\sum_{i, j=1}^{m n} a_{i j}=2(m+1) n-2(n-1) m=2(m+n) .
$$


Example 2.3. For $m=n=3$, problem $\left(E_{\lambda}^{f}\right)$ takes the form

$$
\left\{\begin{array}{l}
4 u(1,1)-u(2,1)-u(1,2)=\lambda f((1,1), u(1,1)) \\
-u(1,1)+4 u(2,1)-u(3,1)-u(2,2)=\lambda f((2,1), u(2,1)) \\
-u(2,1)+4 u(3,1)-u(3,2)=\lambda f((3,1), u(3,1)) \\
-u(1,1)+4 u(1,2)-u(2,2)-u(1,3)=\lambda f((1,2), u(1,2)) \\
-u(2,1)-u(1,2)+4 u(2,2)-u(3,2)-u(2,3)=\lambda f((2,2), u(2,2)) \\
-u(3,1)-u(2,2)+4 u(3,2)-u(3,3)=\lambda f((3,2), u(3,2)) \\
-u(1,2)+4 u(1,3)-u(2,3)=\lambda f((1,3), u(1,3)) \\
-u(2,2)-u(1,3)+4 u(2,3)-u(3,3)=\lambda f((2,3), u(2,3)) \\
-u(3,2)-u(2,3)+4 u(3,3)=\lambda f((3,3), u(3,3))
\end{array}\right.
$$

and, through the map $h$, it can be also written as follows

$$
\left(S_{A, \lambda}^{g}\right) \quad\left\{\begin{array}{l}
4 w_{1}-w_{2}-w_{4}=\lambda g_{1}\left(w_{1}\right) \\
-w_{1}+4 w_{2}-w_{3}-w_{5}=\lambda g_{2}\left(w_{2}\right) \\
-w_{2}+4 w_{3}-w_{6}=\lambda g_{3}\left(w_{3}\right) \\
-w_{1}+4 w_{4}-w_{5}-w_{7}=\lambda g_{4}\left(w_{4}\right) \\
-w_{2}-w_{4}+4 w_{5}-w_{6}-w_{8}=\lambda g_{5}\left(w_{5}\right) \\
-w_{3}-w_{5}+4 w_{6}-w_{9}=\lambda g_{6}\left(w_{6}\right) \\
-w_{4}+4 w_{7}-w_{8}=\lambda g_{7}\left(w_{7}\right) \\
-w_{5}-w_{7}+4 w_{8}-w_{9}=\lambda g_{8}\left(w_{8}\right) \\
-w_{6}-w_{8}+4 w_{9}=\lambda g_{9}\left(w_{9}\right)
\end{array}\right.
$$

where $w_{k}:=u\left(h^{-1}(k)\right), g_{k}\left(w_{k}\right):=f\left(h^{-1}(k), w_{k}\right), \forall k \in[1,9]$, and $A=\left(\begin{array}{ccc}L & -I & 0 \\ -I & L & -I \\ 0 & -I & L\end{array}\right)$.

Furthermore, the matrix $A$ is positive-definite, see [10]. By denoting with $\lambda_{1}, \ldots, \lambda_{m n}$ its eigenvalues such that they satisfy the ordering $0<\lambda_{1}<\lambda_{2} \leq \ldots \leq \lambda_{m n}$, it results, for every $w \in X$,

$$
\lambda_{1}\|w\|^{2} \leq w^{t} A w \leq \lambda_{m n}\|w\|^{2}
$$

and

$$
\|w\|_{\infty} \leq \frac{1}{\sqrt{\lambda_{1}}}\left(w^{t} A w\right)^{1 / 2}
$$

where $\|w\|_{\infty}:=\max _{k \in[1, m n]}\left|w_{k}\right|$.

It is easy to affirm that the solutions of $\left(E_{\lambda}^{f}\right)$ are the critical points of the $C^{1}$-functional

$$
J_{\lambda}(w):=\frac{w^{t} A w}{2}-\lambda \sum_{k=1}^{m n} \int_{0}^{w_{k}} g_{k}(t) d t, \quad \forall w \in X .
$$

Indeed, a column vector $\bar{w}=\left(\bar{w}_{1}, \ldots, \bar{w}_{m n}\right)^{t} \in X$ is a critical point of the functional $J_{\lambda}$ if the gradient of $J_{\lambda}$ at $\bar{w}$ is zero. Moreover, it is well-known that

$$
\frac{\partial w^{t} A w}{\partial w_{k}}=2(A w)_{k}
$$


where $(A w)_{k}:=\sum_{j=1}^{m n} a_{k j} w_{j}$. Thus,

$$
\frac{\partial J_{\lambda}(w)}{\partial w_{k}}=(A w)_{k}-\lambda g_{k}\left(w_{k}\right)
$$

which yields our conclusion.

From now on, set

$$
\Phi(w):=\frac{w^{t} A w}{2}, \text { and } \Psi(w):=\sum_{k=1}^{m n} F_{k}\left(w_{k}\right),
$$

where $F_{k}(t):=\int_{0}^{t} g_{k}(s) d s$, for every $t \in \mathbb{R}$ and $k \in[1, m n]$.

Recall the following strong maximum principle for the problem $\left(E_{\lambda}^{f}\right)$ as given in [9, Proposition 2.2].

Proposition 2.4. Assume that there exists $\bar{u}:[0, m+1] \times[0, n+1] \rightarrow \mathbb{R}$ such that

$$
[\bar{u}(i+1, j)-2 \bar{u}(i, j)+\bar{u}(i-1, j)]+[\bar{u}(i, j+1)-2 \bar{u}(i, j)+\bar{u}(i, j-1)] \leq 0,
$$

for every $(i, j) \in[1, m] \times[1, n]$ and

$$
\bar{u}(i, 0)=\bar{u}(i, n+1)=0, \quad \forall i \in[1, m] ; \quad \bar{u}(0, j)=\bar{u}(m+1, j)=0, \quad \forall j \in[1, n] .
$$

Then, either $\bar{u}$ is the identically zero function or $\bar{u}(i, j)>0$ for every $(i, j) \in[1, m] \times[1, n]$.

Note that, when $f:[1, m] \times[1, n] \times \mathbb{R} \rightarrow \mathbb{R}$ is a nonnegative function, the above maximum principle ensures that every solution of the problem $\left(E_{\lambda}^{f}\right)$ is either zero or positive.

Remark 2.5. (Contemplate $\left(E_{\lambda}^{f}\right)$ as a discrete nonlinear Laplace 2-dimensional equation) As pointed out in [7], problem $\left(E_{\lambda}^{f}\right)$ serves as the discrete counterpart of the following problem

$$
\left\{\begin{array}{l}
\frac{\partial^{2} u}{\partial x^{2}}+\frac{\partial^{2} u}{\partial y^{2}}+\lambda f((x, y), u(x, y))=0 \\
u(x, 0)=u(x, n+1)=0, \forall x \in(0, m+1) \\
u(0, y)=u(m+1, y)=0, \forall y \in(0, n+1)
\end{array}\right.
$$

However, the obtained results cannot be directly achieved proving the existence of solutions for the above equation.

Remark 2.6. (Interpretation and consequences of the multi-solutions)

Problem $\left(E_{\lambda}^{f}\right)$ can be expressed starting from the following algebraic terminology.

A lattice point $z:=(i, j)$ in the plane is a point of integer coordinates. Two lattice points are said neighbours if their Euclidean distance is 1 . An edge (resp. directed edge) is a pair (resp. an ordered pair) $\left\{z, z^{\star}\right\}$ of neighbouring points. A path between two lattice points $z$ and $z^{\star}$ is a sequence $z=z_{0}, \ldots, z_{s}=z^{\star}$ of lattice points such that $z_{i}$ and $z_{i+1}$ are neighbours for $0 \leq i \leq s-1$. A set $S$ of lattice points is said to be connected if there is a path contained in $S$ between any two points of $S$. A net is a finite and connected set of lattice points. An exterior boundary point of a net $S$ is a point outside $S$ but having a neighbour in $S$. The set of all edges (resp. directed edges) of a net $S$ is denoted by $\Gamma(S)$ (resp. $E(S)$ ). The pair $(S, \Gamma(S))$ is a planar graph, and the pair $(S, E(S))$ is a directed planar graph. Let $\partial S$ be the set of all exterior boundary points. 
With the above notations, problem $\left(E_{\lambda}^{f}\right)$ can be rewritten as follows

$$
\begin{cases}D u(z)+\lambda f(z, u(z))=0, & z \in S \\ u(z)=0, & z \in \partial S\end{cases}
$$

where

$$
D u(z):=[u(i+1, j)-2 u(i, j)+u(i-1, j)]+[u(i, j+1)-2 u(i, j)+u(i, j-1)],
$$

is the discrete Laplacian acting on a function $u: S \cup \partial S \rightarrow \mathbb{R}$.

A nice application can be found studying connectness between nets in networks and telecommunication phenomena, see [25].

\section{Main Results}

For a given nonnegative constant $c$ and a positive constant $d$, with

$$
\frac{c^{2}}{2} \lambda_{1} \neq(m+n) d^{2}
$$

put

$$
a_{d}(c):=\frac{\sum_{k=1}^{m n}\left(\max _{|t| \leq c} F_{k}(t)\right)-\sum_{k=1}^{m n} F_{k}(d)}{\frac{c^{2}}{2} \lambda_{1}-(m+n) d^{2}} .
$$

We formulate our main result as follows.

Theorem 3.1. Assume that there exist a nonnegative constant $c_{1}$ and two positive constants $c_{2}$ and $d$ with $c_{1}<\sqrt{\frac{2}{\lambda_{1}}(m+n)} d<c_{2}$ such that

(a $\left.a_{1}\right) \quad a_{d}\left(c_{2}\right)<a_{d}\left(c_{1}\right)$.

Then, for each $\lambda \in] \frac{1}{a_{d}\left(c_{1}\right)}, \frac{1}{a_{d}\left(c_{2}\right)}\left[\right.$, problem $\left(E_{\lambda}^{f}\right)$ admits at least one nontrivial solution $u_{1} \in X$ such that $c_{1}^{2} \lambda_{1}<u_{1}^{t} A u_{1}<c_{2}^{2} \lambda_{1}$.

Proof. Fix $\lambda$ as in the conclusion, and take the real Banach space $X$ as defined in Section 2, and put $\Phi$ and $\Psi$ as in (2.5) and $J_{\lambda}$ as in (2.4). Our aim is to apply Lemma 2.1. We observe that Assumption (A) holds. Indeed, we clearly see that the functionals $\Phi, \Psi$ are $C^{1}$ on $X$, and inequality (2.2) yields $\Phi$ is coercive. Choose

$$
w(t)=d \text { for every } t \in[0, T]
$$

as well as $r_{1}:=\frac{c_{1}^{2}}{2} \lambda_{1}$ and $r_{2}:=\frac{c_{2}^{2}}{2} \lambda_{1}$. By using the condition

$$
c_{1}<\sqrt{\frac{2}{\lambda_{1}}(m+n)} d<c_{2}
$$

we get $r_{1}<\Phi(w)<r_{2}$. Thanks to (2.3) and

$$
\left\{w \in X: w^{t} A w<2 r_{2}\right\} \subset\left\{w \in X:\left|w_{k}\right| \leq c_{2}, \forall k \in[1, m n]\right\},
$$

it follows that

$$
\sup _{u \in \Phi^{-1}\left(-\infty, r_{2}\right)} \Psi(u) \leq \sum_{k=1}^{m n} \max _{|t| \leq c_{2}} F_{k}(t) .
$$


Therefore, one has

$$
\begin{aligned}
\vartheta\left(r_{1}, r_{2}\right) & \leq \frac{\sup _{u \in \Phi^{-1}\left(-\infty, r_{2}\right)} \Psi(u)-\Psi(w)}{r_{2}-\Phi(w)} \\
& \leq \frac{\sum_{k=1}^{m n} \max _{|t| \leq c_{2}} F_{k}(t)-\sum_{k=1}^{m n} F_{k}(d)}{\frac{c_{2}^{2}}{2} \lambda_{1}-(m+n) d^{2}} \\
& =a_{d}\left(c_{2}\right) .
\end{aligned}
$$

On the other hand, arguing as before, one has

$$
\begin{aligned}
\rho\left(r_{1}, r_{2}\right) & \geq \frac{\Psi(w)-\sup _{u \in \Phi^{-1}\left(-\infty, r_{1}\right]} \Psi(u)}{\Phi(w)-r_{1}} \\
& \geq \frac{\sum_{k=1}^{m n} F_{k}(d)-\sum_{k=1}^{m n} \max _{|t| \leq c_{1}} F_{k}(t)}{(m+n) d^{2}-\frac{c_{1}^{2}}{2} \lambda_{1}} \\
& =a_{d}\left(c_{1}\right) .
\end{aligned}
$$

Hence, from Assumption $\left(\mathrm{a}_{1}\right)$, one has $\vartheta\left(r_{1}, r_{2}\right)<\rho\left(r_{1}, r_{2}\right)$. Therefore, employing Lemma 2.1, for each

$$
\lambda \in] \frac{1}{a_{d}\left(c_{1}\right)}, \frac{1}{a_{d}\left(c_{2}\right)}[
$$

the functional $J_{\lambda}$ admits at least one critical point $u_{1} \in X$ such that $r_{1}<\Phi\left(u_{1}\right)<r_{2}$, that is

$$
c_{1}^{2} \lambda_{1}<u_{1}^{t} A u_{1}<c_{2}^{2} \lambda_{1}
$$

The proof is complete.

The following result is an immediate consequence of Theorem 3.1.

Corollary 3.2. Assume that there exist two positive constants $c$ and $d$ with $\sqrt{\frac{2}{\lambda_{1}}(m+n)} d<c$ such that (a) $\quad \frac{\sum_{k=1}^{m n}\left(\max _{|t| \leq c} F_{k}(t)\right)}{c^{2}}<\frac{\lambda_{1}}{2(m+n)} \frac{\sum_{k=1}^{m n} F_{k}(d)}{d^{2}}$.

Then, for every $\lambda \in] \frac{(m+n) d^{2}}{\sum_{k=1}^{m n} F_{k}(d)}, \frac{\frac{c^{2}}{2} \lambda_{1}}{\sum_{k=1}^{m n}\left(\max _{|t| \leq c} F_{k}(t)\right)}\left[\right.$, the problem $\left(E_{\lambda}^{f}\right)$ admits at least one nontrivial solution $u_{1} \in X$ such that $0<u_{1}^{t} A u_{1}<c^{2} \lambda_{1}$.

Proof. The conclusion follows from Theorem 3.1 by taking $c_{1}=0$ and $c_{2}=c$. Indeed, from the assumptions, one has

$$
\begin{aligned}
a_{d}(c) & <\frac{\left(1-\frac{(m+n) d^{2}}{\frac{c_{1}^{2}}{2} \lambda_{1}}\right) \sum_{k=1}^{m n}\left(\max _{|t| \leq c} F_{k}(t)\right)}{\frac{c^{2}}{2} \lambda_{1}-(m+n) d^{2}} \\
& =\frac{\sum_{k=1}^{m n}\left(\max _{|t| \leq c} F_{k}(t)\right)}{\frac{c^{2}}{2} \lambda_{1}} \\
& <\frac{\sum_{k=1}^{m n} F_{k}(d)}{\left.(m+n) d^{2}\right)} \\
& =a_{d}(0) .
\end{aligned}
$$


In particular, one has

which follows

$$
a_{d}(c)<\frac{\sum_{k=1}^{m n}\left(\max _{|t| \leq c} F_{k}(t)\right)}{\frac{c^{2}}{2} \lambda_{1}}
$$

$$
\frac{\frac{c^{2}}{2} \lambda_{1}}{\sum_{k=1}^{m n}\left(\max _{|t| \leq c} F_{k}(t)\right)}<\frac{1}{a_{d}(c)} .
$$

Hence, Theorem 3.1, ensures the result.

Now, we present an application of Lemma 2.1, which we will use to obtain multiple solutions.

Theorem 3.3. Assume that there exist two positive constants $\bar{c}$ and $\bar{d}$ with $\bar{c}<\sqrt{\frac{2(m+n)}{\lambda_{1}}} \bar{d}$ such that

(b 1$) \quad \sum_{k=1}^{m n}\left(\max _{|t| \leq \bar{c}} F_{k}(t)\right)<\sum_{k=1}^{m n} F_{k}(\bar{d})$

$\left(\mathrm{b}_{2}\right) \quad \limsup _{t \rightarrow+\infty} \frac{F_{k}(t)}{t^{2}}<\frac{1}{\bar{\lambda}}, \forall k \in[1, m n]$, where

$$
\bar{\lambda}=\frac{(m+n) \bar{d}^{2}-\frac{\bar{c}^{2}}{2} \lambda_{1}}{\sum_{k=1}^{m n} F_{k}(\bar{d})-\sum_{k=1}^{m n}\left(\max _{|t| \leq \bar{c}} F_{k}(t)\right)} .
$$

Then, for each $\lambda>\bar{\lambda}$, problem $\left(E_{\lambda}^{f}\right)$ admits at least one nontrivial solution $\overline{u_{1}} \in X$ such that $\overline{u_{1}}{ }^{t} A \overline{u_{1}}>$ $\bar{c}^{2} \lambda_{1}$.

Proof. Fix $\lambda>\bar{\lambda}$, take the real Banach space $X$ as defined in Section 2, and put $\Phi$ and $\Psi$ as in (2.5) and $J_{\lambda}$ as in (2.4). Our aim is to apply Lemma 2.1. Moreover, from $\left(b_{2}\right)$ there exist two constants $\gamma, \eta \in \mathbb{R}$ with

such that

$$
0<\gamma<\frac{1}{\bar{\lambda}}
$$

$$
\frac{1}{\lambda_{1}} F_{k}(t) \leq \gamma t^{2}+\eta \text { for all } t \in \mathbb{R}
$$

for all $k \in[1, m n]$. Fix $u=\left(u_{1}, \cdots, u_{m n}\right) \in X$. Then

$$
\sum_{k=1}^{m n} F_{k}\left(u_{k}\right) \leq \lambda_{1}\left(\gamma \sum_{k=1}^{m n}\left|u_{k}\right|^{2}+\eta\right) .
$$

So, for any fixed $\lambda>\bar{\lambda}$, we have from (2.2)-(3.2) that

$$
\begin{aligned}
\Phi(u)-\lambda \Psi(u) & =\frac{u^{t} A u}{2}-\lambda \sum_{k=1}^{m n} F_{k}\left(u_{k}\right) \\
& \geq \frac{u^{t} A u}{2}-\lambda \lambda_{1} \gamma \sum_{k=1}^{m n}\left|u_{k}\right|^{2}-\lambda \lambda_{1} \eta \\
& \geq \frac{u^{t} A u}{2}-m n \lambda \lambda_{1} \gamma\|u\|_{\infty}^{2}-\lambda \lambda_{1} \eta \\
& \geq \frac{u^{t} A u}{2}-m n \lambda \lambda_{1} \gamma \frac{1}{\lambda_{1}} u^{t} A u-\lambda \lambda_{1} \eta \\
& =\left(\frac{1}{2}-m n \lambda \gamma\right) u^{t} A u-\lambda \lambda_{1} \eta \\
& \geq(1-m n \gamma \bar{\lambda}) \lambda_{1}\|u\|^{2}-\bar{\lambda} \lambda_{1} \eta
\end{aligned}
$$


Since $1-m n \gamma \bar{\lambda}>0$, this leads to the coercivity of the functional $\Phi-\lambda \Psi$. Put $\bar{r}=\frac{\bar{c}^{2}}{2} \lambda_{1}$ and choose $w=\left(w_{1}, \ldots, w_{m n}\right)$ with $w_{k}=\bar{d}$ for every $k \in[1, m n]$. We have $w^{t} A w=2(m+n) \bar{d}^{2}$. Arguing as in the proof of Theorem 3.1, we obtain that

$$
\rho_{2}(\bar{r}) \geq \frac{\sum_{k=1}^{m n} F_{k}(\bar{d})-\sum_{k=1}^{m n}\left(\max _{|t| \leq \bar{c}} F_{k}(t)\right)}{(m+n) \bar{d}^{2}-\frac{\bar{c}^{2}}{2} \lambda_{1}} .
$$

So, from our assumptions, it follows that $\rho_{2}(\bar{r})>0$. Hence, Lemma 2.2 ensures that the functional $\Phi-\lambda \Psi$ admits at least one local minimum $\bar{u} \in X$ such that ${\overline{u_{1}}}^{t} A{\overline{u_{1}}}>\bar{c}^{2} \lambda_{1}$, and since any critical point of the functional $\Phi-\lambda \Psi$ is a solution of $\left(E_{\lambda}^{f}\right)$, we have the conclusion.

Now, we point out some results in which the function $f$ has separated variables. To be precise, consider the discrete system, namely $\left(E_{\lambda}^{z, \alpha}\right)$, given as follows

$$
\begin{gathered}
{[u(i+1, j)-2 u(i, j)+u(i-1, j)]+[u(i, j+1)-2 u(i, j)+u(i, j-1)]} \\
+\lambda \alpha(i, j) z(u(i, j))=0, \quad \forall(i, j) \in[1, m] \times[1, n]
\end{gathered}
$$

with boundary conditions

$$
\begin{gathered}
u(i, 0)=u(i, n+1)=0 \quad \forall i \in[1, m], \\
u(0, j)=u(m+1, j)=0 \quad \forall j \in[1, n],
\end{gathered}
$$

where $\alpha:[1, m] \times[1, n] \rightarrow \mathbb{R}$ is a nonnegative and non-zero function and $z:[0,+\infty) \rightarrow \mathbb{R}$ is a continuous function such that $z(0)=0$.

For a given nonnegative constant $c$ and a positive constant $d$, with

$$
\frac{c^{2}}{2} \lambda_{1} \neq(m+n) d^{2}
$$

put

Setting

$$
b_{d}(c):=\frac{\max _{0 \leq \xi \leq c} \int_{0}^{\xi} z(s) d s-\int_{0}^{d} z(s) d s}{\frac{c^{2}}{2} \lambda_{1}-(m+n) d^{2}} .
$$

$$
f((i, j), t):= \begin{cases}\alpha(i, j) z(t), & \text { if } t \geq 0, \\ 0, & \text { if } t<0,\end{cases}
$$

for every $(i, j) \in[1, m] \times[1, n]$ and $t \in \mathbb{R}$, taking into account Proposition 2.4, we have the following existence results as consequences of Theorem 3.1, Corollary 3.2 and Theorem 3.3, respectively.

Theorem 3.4. Assume that there exist a nonnegative constant $c_{1}$ and two positive constants $c_{2}$ and $d$ with $c_{1}<\sqrt{\frac{2}{\lambda_{1}}(m+n)} d<c_{2}$ such that

Then, for each $\lambda \in] \frac{1}{\sum_{k=1}^{m n} \alpha\left(h^{-1}(k)\right)} \frac{1}{b_{d}\left(c_{1}\right)}, \frac{1}{\sum_{k=1}^{m n} \alpha\left(h^{-1}(k)\right)} \frac{1}{b_{d}\left(c_{2}\right)}\left[\right.$, problem $\left(E_{\lambda}^{z, \alpha}\right)$ admits at least one positive solution $u_{1} \in X$ such that $c_{1}^{2} \lambda_{1}<u_{1}^{t} A u_{1}<c_{2}^{2} \lambda_{1}$.

Corollary 3.5. Assume that there exist two positive constants $c$ and $d$ with $\sqrt{\frac{2}{\lambda_{1}}(m+n)} d<c$ such that 
$\left(\mathrm{a}_{4}\right) \quad \frac{\max _{0 \leq \xi \leq c} \int_{0}^{\xi} z(s) d s}{c^{2}}<\frac{\lambda_{1}}{2(m+n)} \frac{\int_{0}^{d} z(s) d s}{d^{2}}$.

Then, for every $\lambda \in] \frac{1}{\sum_{k=1}^{m n} \alpha\left(h^{-1}(k)\right)} \frac{(m+n) d^{2}}{\int_{0}^{d} z(s) d s}, \frac{1}{\sum_{k=1}^{m n} \alpha\left(h^{-1}(k)\right)} \frac{\frac{c^{2}}{2} \lambda_{1}}{\max _{0 \leq \xi \leq c} \int_{0}^{\xi} z(s) d s}\left[\right.$,problem $\left(E_{\lambda}^{z, \alpha}\right)$ admits at least one positive solution $u_{1} \in X$ such that $0<u_{1}^{t} A u_{1}<c^{2} \lambda_{1}$.

Theorem 3.6. Assume that there exist two positive constants $\bar{c}$ and $\bar{d}$ with $\bar{c}<\sqrt{\frac{2(m+n)}{\lambda_{1}}} \bar{d}$, such that

$\left(\mathrm{b}_{3}\right) \max _{0 \leq \xi \leq \bar{c}} \int_{0}^{\xi} z(s) d s<\int_{0}^{\bar{d}} z(s) d s$;

(b) $\quad \lim \sup _{t \rightarrow+\infty} \frac{\int_{0}^{t} z(s) d s}{t^{2}}<\frac{1}{\lambda^{\prime}}$.

Then, for each $\lambda>\overline{\lambda^{\prime}}$, where

$$
\overline{\lambda^{\prime}}=\frac{1}{\sum_{k=1}^{m n} \alpha\left(h^{-1}(k)\right)} \frac{(m+n) \bar{d}^{2}-\frac{\bar{c}^{2}}{2} \lambda_{1}}{\int_{0}^{\bar{d}} z(s) d s-\max _{0 \leq \xi \leq \bar{c}} \int_{0}^{\xi} z(s) d s},
$$

problem $\left(E_{\lambda}^{z, \alpha}\right)$ admits at least one positive solution $\overline{u_{1}} \in X$ such that ${\overline{u_{1}}}^{t} A \overline{u_{1}}>\bar{c}^{2} \lambda_{1}$.

Here we illustrate Theorem 3.6 by giving the following example.

Example 3.7. Consider the problem

$$
\begin{gathered}
{[u(i+1, j)-2 u(i, j)+u(i-1, j)]+[u(i, j+1)-2 u(i, j)+u(i, j-1)]} \\
+\lambda z(u(i, j))=0, \quad \forall(i, j) \in[1,2] \times[1,2]
\end{gathered}
$$

with boundary conditions

$$
\begin{gathered}
u(i, 0)=u(i, 3)=0, \quad \forall i \in[1,2], \\
u(0, j)=u(3, j)=0, \quad \forall j \in[1,2],
\end{gathered}
$$

where $z(t)=e^{-t} t^{2}(3-t)$ for each $t \geq 0$. The matrix $A$ associated to the above problem is

$$
A=\left(\begin{array}{ccccc}
4 & -1 & \vdots & -1 & 0 \\
-1 & 4 & \vdots & 0 & -1 \\
\ldots & \ldots & \ldots & \ldots & \ldots \\
-1 & 0 & \vdots & 4 & -1 \\
0 & -1 & \vdots & -1 & 4
\end{array}\right) .
$$

Clearly, its characteristic polynomial can be easily computed obtaining that

$$
p(\lambda):=\operatorname{det}\left(A-\lambda I_{4}\right)=\lambda^{4}-16 \lambda^{3}+92 \lambda^{2}-224 \lambda+192=(\lambda-2)(\lambda-4)^{2}(\lambda-6) .
$$

Therefore, the first eigenvalue $\lambda_{1}$ is equal to 2 . By choosing $\bar{c}=1$ and $\bar{d}=2$, since

$$
\limsup _{t \rightarrow+\infty} \frac{\int_{0}^{t} z(s) d s}{t^{2}}=0
$$

we observe that all hypotheses of Theorem 3.6 are fulfilled. Hence, by applying Theorem 3.6, for every $\lambda>\frac{15}{4\left(8 e^{-2}-e^{-1}\right)}$ the above problem admits at least one positive solution $\overline{u_{1}} \in X$ such that $\overline{u_{1}}{ }^{t} A \overline{u_{1}}>2$. 
We give a special case of Corollary 3.5 as follows.

Theorem 3.8. Assume that

$$
\lim _{t \rightarrow 0^{+}} \frac{z(t)}{t}=+\infty
$$

Then, for each $\lambda \in] 0, \frac{1}{\sum_{k=1}^{m n} \alpha\left(h^{-1}(k)\right)} \sup _{c>0} \frac{\frac{c^{2}}{2} \lambda_{1}}{\max _{0 \leq \xi \leq c} \int_{0}^{\xi} z(s) d s}\left[\right.$, problem $\left(E_{\lambda}^{z, \alpha}\right)$ admits at least one positive solution in $X$.

Proof. For fixed

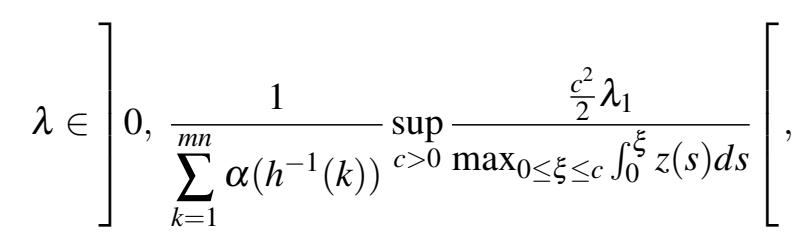

there exists a positive constant $c$ such that

$$
\lambda<\frac{1}{\sum_{k=1}^{m n} \alpha\left(h^{-1}(k)\right)} \frac{\frac{c^{2}}{2} \lambda_{1}}{\max _{0 \leq \xi \leq c} \int_{0}^{\xi} z(s) d s} .
$$

Moreover, the condition

$$
\lim _{t \rightarrow 0^{+}} \frac{z(t)}{t}=+\infty
$$

yields

$$
\lim _{t \rightarrow 0^{+}} \frac{\int_{0}^{t} z(s) d s}{t^{2}}=+\infty .
$$

Therefore, we can choose positive constant $d$ satisfying

such that

$$
\sqrt{\frac{2}{\lambda_{1}}(m+n)} d<c
$$

$$
\frac{\lambda(m+n)}{\sum_{k=1}^{m n} \alpha\left(h^{-1}(k)\right)}<\frac{\int_{0}^{d} z(s) d s}{d^{2}} .
$$

Hence, from Corollary 3.5 we have the conclusion.

We end this paper by presenting the following example to illustrate Theorem 3.8.

Example 3.9. Consider the problem

$$
\begin{gathered}
{[u(i+1, j)-2 u(i, j)+u(i-1, j)]+[u(i, j+1)-2 u(i, j)+u(i, j-1)]} \\
+\lambda z(u(i, j))=0, \quad \forall(i, j) \in[1,2] \times[1,2]
\end{gathered}
$$

with boundary conditions

$$
\begin{aligned}
& u(i, 0)=u(i, 3)=0, \quad \forall i \in[1,2], \\
& u(0, j)=u(3, j)=0, \quad \forall j \in[1,2]
\end{aligned}
$$

where

$$
z(t)= \begin{cases}0, & t=0 \\ 1+t \sin ^{2}(\ln (t)), & t>0\end{cases}
$$


It is clear that $\lim _{t \rightarrow 0^{+}} \frac{z(t)}{t}=+\infty$. The first eigenvalue $\lambda_{1}$ of the matrix $A$ associated to the above problem is equal to 2 (see Example 3.7). Hence, by applying Theorem 3.8, for every

$$
\lambda \in] 0, \frac{1}{4} \sup _{c>0} \frac{c^{2}}{c+\frac{1}{2} c^{2}\left(\sin ^{2}(\ln c)-\frac{1}{4} \sin (2 \ln c)+\frac{1}{4} \cos (2 \ln c)\right)}[,
$$

the above problem admits at least one positive solution in $X$.

\section{REFERENCES}

[1] G. Zhang, L. Bai, Existence of solutions for a nonlinear algebraic system, Discrete Dyn. Nat. Soc. 2009 (20009), Article ID 785068.

[2] R. P. Agarwal, K. Perera, D. O'Regan, Multiple positive solutions of singular discrete $p$-Laplacian problems via variational methods, Adv. Difference Equ. 2 (2005), 93-99.

[3] C. Bereanu, J. Mawhin, Existence and multiplicity results for nonlinear second order difference equations with Dirichlet boundary conditions, Math. Bohem. 131 (2006), 145-160.

[4] P. Candito, G. Molica Bisci, Existence of two solutions for a nonlinear second-order discrete boundary value problem, Adv. Nonlinear Stud. 11 (2011), 443-453.

[5] J. Chu, D. Jiang, Eingenvalues and discrete boundary value problems for the one-dimensional $p$-Laplacian, J. Math. Anal. Appl. 305 (2005), 452-465.

[6] G. Zhang, S. Liu, On a class of semipositone discrete boundary value problems, J. Math. Anal. Appl. 325 (2007), $175-182$.

[7] M. Galewski, A. Orpel, On the existence of solutions for discrete elliptic boundary value problems, Appl. Anal. 8 (2010), 1879-1891.

[8] S. Heidarkhani, M. Imbesi, Multiple solutions for partial discrete Dirichlet problems depending on a real parameter, J. Difference Equ. Appl. 21, 2 (2015), 96-110.

[9] M. Imbesi, G. Molica Bisci, Discrete elliptic Dirichlet problems and nonlinear algebraic aystems, Mediterr. J. Math. 13 (2016), 263-278.

[10] J. Ji, B. Yang, Eigenvalue comparisons for boundary value problems of the discrete elliptic equation, Commun. Appl. Anal. 12 (2008), 189-197.

[11] G. Molica Bisci, D. Repov̌̌, Nonlinear algebraic systems with discontinuous terms, J. Math. Anal. Appl. 398 (2013), 846-856.

[12] Y. Yang, J. Zhang, Existence results for a nonlinear system with a parameter, J. Math. Anal. Appl. 340 (2008), 658-668.

[13] Y. Yang, J. Zhang, Existence and multiple solutions for a nonlinear system with a parameter, Nonlinear Anal. 70 (2009), 2542-2548.

[14] G. Zhang, Existence of non-zero solutions for a nonlinear system with a parameter, Nonlinear Anal. 66 (2007), $1410-1416$.

[15] G. Zhang, S. S. Cheng, Existence of solutions for a nonlinear algebraic system with a parameter, J. Math. Anal. Appl. 314 (2006), 311-319.

[16] G. Zhang, W. Feng, On the number of positive solutions of a nonlinear algebraic system, Linear Algebra Appl. 422 (2007), 404-421.

[17] R. P. Agarwal, Difference Equations and Inequalities: theory, methods and applications, Marcel Dekker, New York-Basel, 2000.

[18] W. G. Kelly, A. C. Peterson, Difference Equations, An introduction with applications, Academic Press, San Diegol New York, 1991.

[19] A. Kristály, M. Mihăilescu, V. Rădulescu, Discrete boundary value problems involving oscillatory nonlinearities: small and large solutions, J. Difference Equ. Appl. 17 (2011), 1431-1440.

[20] A. Kristály, M. Mihăilescu, V. Rădulescu, S. Tersian, Spectral estimates for a nonhomogeneous difference problem, Commun. Contemp. Math. 12 (2010), 1015-1029.

[21] A. Cabada, A. Iannizzotto, S. Tersian, Multiple solutions for discrete boundary value problems, J. Math. Anal. Appl. 356 (2009), 418-428.

[22] G. Bonanno, G. Molica Bisci, Infinitely many solutions for a boundary value problem with discontinuous nonlinearities, Bound. Value Probl. 2009 (2009), Art. ID 670675. 
[23] G. Bonanno, A critical point theorem via the Ekeland variational principle, Nonlinear Anal. 75 (2012), 2992-3007.

[24] P. Candito, G. Molica Bisci, Existence of solutions for a nonlinear algebraic system with parameter, Appl. Math. Comput. 218 (2012), 11700-11707.

[25] S.S. Cheng, Partial Difference Equations, Taylor \& Francis, London, 2003.

[26] B. Ricceri, A general variational principle and some of its applications, J. Comput. Appl. Math. 113 (2000), $401-410$. 